\title{
Potential inhibitors of SARS-CoV-2 Main protease $\left(\mathrm{M}^{\mathrm{pro}}\right)$ identified from the library of FDA approved drugs using molecular docking studies
}

\author{
Dipesh Kumar Verma ${ }^{1}$, Srajan Kapoor ${ }^{1}$, Satyajeet Das ${ }^{1}$ and Krishan Gopal Thakur ${ }^{1 \#}$ \\ ${ }^{1}$ Structural Biology Laboratory, GNR Protein Centre, Council of Scientific and Industrial Research-Institute of \\ Microbial Technology (CSIR-IMTECH), Chandigarh-160036, India.
}

\section{Correspondence}

${ }^{\#}$ Krishan Gopal Thakur

Email: krishang@imtech.res.in

Mob. No: +91 8427717723

\begin{abstract}
Corona Virus Infectious Disease-2019 (COVID-19) outbreak originated recently at Wuhan, China in December 2019. It has already spread rapidly to more than 200 countries and has been declared a pandemic by WHO. It is caused by a beta-coronavirus named as SARS-CoV-2. There is no definitive cure, either drug or vaccine, to treat or prevent this viral disease. Recently, the crystal structure of the main protease $\mathbf{M}^{\text {pro }}$ has been determined. $\mathbf{M}^{\text {pro }}$ is responsible for the proteolytic maturation of the polyprotein essential for the viral replication and transcription, which makes it an important drug target. The discovery of new drug molecules may take years before getting to the clinics. So, considering urgency we performed molecular docking studies using FDA approved drugs to identify molecules that could potentially bind to the substrate-binding site and inhibit SARS-CoV-2 main protease $\left(\mathrm{M}^{\mathrm{pro}}\right)$. We used the Glide module in Schrodinger software suite to perform molecular docking studies followed by MM-GBSA based energy calculations to score the hit molecules. Molecular docking and manual analysis suggest that several drugs may bind and potentially inhibit $\mathrm{M}^{\text {pro }}$. We also performed molecular simulations studies for selected compounds to evaluate protein-drug interactions. Interestingly, we observed only one antiviral compound, Adefovir, in the top50 list of compounds. Considering bioavailability, lesser toxicity, route of administration some of the top-ranked drugs including lumefantrine (antimalarial), dipyridamole (coronary vasodilator), dihydroergotamine (used for treating migraine), hexoprenaline (antiasthmatic), riboflavin (vitamin B2) and pantethine (vitamin B5) may be taken forward for further in vitro and in vivo experiments to investigate their therapeutic potential.
\end{abstract}

Keywords: COVID-19, SARS-CoV-2, Mro, Molecular docking, MM-GBSA 


\section{Introduction}

Coronaviruses are a group of RNA viruses that cause diseases in mammals and birds. The outbreaks of Severe Acute Respiratory Syndrome (SARS) (2003), Middle East Respiratory Syndrome (MERS) (2012) and the recent outbreak of COVID-19 (SARS-CoV-2) have shown the immense potential of these viruses to infect humans causing deaths and large economic losses. Although the fatality rate of COVID-19 $(\sim 4 \%)$ is lower compared to SARS $(\sim 10 \%)$ and MERS $(\sim 35 \%)$, the rate of its spreading is much faster than both of these (Guo et al., 2020). Till date, more than 1 million people have been infected worldwide and more than 50,000 people have already died in last three months. COVID-19 infection can cause symptoms ranging from mildcold like symptoms to severe illness with pneumonia, respiratory problems, and death (Mackenzie and Smith, 2020; Schoeman and Fielding, 2019). Several countries have imposed lockdown which is helping in restricting the spread of the disease, however it has not been completely successful. Besides loss of human lives, COVID-19 is causing severe economic losses to both developed and developing countries.

The coronavirus enters the cell with the help of its trimeric glycosylated spike (S) protein, a class I viral fusion protein with two subunits $\mathrm{S} 1$ and $\mathrm{S} 2$. A fusion peptide is located between the $\mathrm{N}$ - and the C-terminal regions of S2 (Walls et al., 2017). The S1 subunit binds with the angiotensinconverting enzyme-2 (Ace-2) followed by S2 domain and fusion peptide mediated fusion of the viral envelope and cell membrane (Hoffmann et al., 2020). Proteolytic cleavage of S protein after binding with Ace-2 by various host cell proteases like Transmembrane Serine Protease 2 (TMPRSS2), endosomal cathepsins, etc. causes the fusion of the viral envelope to the host cell and delivers the viral nucleocapsid into the host cell (Hoffmann et al., 2020; Millet and Whittaker, 2015; Simmons et al., 2005). The released genomic RNA of the virus is then recognized by the host cell translation machinery which synthesized the polyprotein 1a (pp1a) and 1ab (pp1ab) by ribosomal frame shifting (Brierley et al., 1987). Then the proteolytic cleavage of the polyproteins by main protease $\left(\mathrm{M}^{\text {pro }}\right)$ produces various non-structural proteins (nsp) (Li, 2016). It has been shown previously that the $\mathrm{nsp} 5\left(\mathrm{M}^{\mathrm{pro}}\right)$ of porcine coronavirus can also modify the key players of the host immune system by mediating the cleavage of NFKB, STAT-2, therefore, affecting the production of IFN- $\beta$ and expression of interferon-stimulated genes (Koonpaew et al., 2019).

Currently, there are many drug candidates in clinical use or undergoing clinical trials worldwide to treat COVID-19. For example, poly-ADP-ribose polymerase 1 (PARP1) inhibitor, CVL218 is in phase 1 clinical trial (Ge et al., 2020). Remdesivir, which was originally developed for the treatment of the Ebola outbreak, was given to a patient in the United States causing the recovery of a patient from the severely ill category (Al-Tawfiq et al., 2020; Gao et al., 2020). 
Limited clinical studies on two anti-malarial drugs, chloroquine and hydroxychloroquine, have shown potential for treating COVID-19 (Khaerunnisa and Soetjipto, 2020). However, the toxicity associated with the use of these drugs have also been reported (McChesney, 1983). The national medical products administration of China has approved Flvilavir, for the treatment of coronavirus (https://www.clinicaltrialsarena.com/analysis/coronavirus-mers-cov-drugs/). Several vaccines for coronavirus are also under clinical trials and over 40 vaccines are under different stages of development (https://www.clinicaltrialsarena.com/analysis/coronavirus-mers-cov-drugs/).

Coronavirus $\mathrm{M}^{\text {pro }}$ is a dimeric protein that cleaves the polyprotein into several functional proteins helping in viral replication and transcription (Xue et al., 2008). Hence, $M^{\text {pro }}$ is an important drug target for treating COVID-19 (Prajapat et al., 2020). $\mathrm{M}^{\text {pro }}$ is a three-domain protein comprising of domains I, II and III. Domain I and II have a chymotrypsin-like fold (Xue et al., 2008). The substrate-binding cleft of $M^{\text {pro }}$ is located between domains I and II. $M^{\text {pro }}$ is not proteolytically active in the monomeric form because the substrate-binding site is not well organized while in the dimeric form the substrate-binding site adopts proper conformation (Li, 2016).

Here, using the Schrodinger software suite, we screened the FDA approved drug library against SARS-CoV-2 $\mathrm{M}^{\text {pro }}$ to search for the drugs that can be potentially used for treating COVID19 pandemic. Our data suggest that several drugs could potentially bind and inhibit $\mathbf{M}^{\text {pro }}$ activity. Considering safety profile and bioavailability, drugs among the top hits that could be taken further for in vitro and in vivo studies include lumefantrine, dipyridamole, dihydroergotamine, hexoprenaline, and riboflavin as potential candidates for treating COVID-19.

\section{Results}

\section{Sequence and structural comparison of $M^{\text {pro }}$}

The structural analysis of the protein inhibitor complex (PDB ID: 6LU7) revealed that covalent inhibitor N3 binds at the hydrophobic ligand binding pocket and interacts with His163, His164, Glu166, Gln189, and Thr190 residues of $\mathbf{M}^{\text {pro }}$ (Jin et al., 2020). Multiple sequence alignment and ConSurf analyses suggest that this ligand binding site is highly conserved among the members of coronavirus family (Figure 1 and 2) (Ashkenazy et al., 2010). In MERS and H-CoV N142C mutation was observed while, in MERS H-CoV, IBV and PEDV, H164Q mutation was observed. Also, in MERS and Bat-CoV T191V mutation was observed. At the position 215 Bat-CoV has a stretch of residues i.e. VKESSF which is absent in all other coronaviruses. We also observed 
insertion of H247, V248 and E270 in both SARS-CoV-2 and SARS-CoV. These residues are absent in the other coronaviruses analyzed in this study (Figure 1).

\section{In silico screening of FDA approved drugs to identify potential binders}

The FDA approved drug library and receptor preparation for molecular docking studies were carried out using Schrodinger Suite, 2019 using protein preparation wizard and LigPrep modules (Chen and Foloppe, 2010). The potential drug binding sites in the crystal structure were identified using the SiteMap (Halgren, 2007). SiteMap analysis suggested five possible druggable sites for ligand binding with site scores between 0.549-0.943. The top binding site with site score of 0.943 was selected for grid generation followed by docking. This site corresponds to the highly conserved substrate binding pocket in coronaviruses. The molecular docking was performed using Glide Xtra-Precision (XP) module (Arowosegbe et al., 2019). Glide XP uses an anchor-and-grow approach for sampling and E-model scoring function to select between protein-ligand complexes of a given ligand and the GlideScore (Halgren et al., 2004). The XP docked structures were further used for binding free-energy calculations using the Molecular Mechanics energies combined with the Generalized Born and Surface Area continuum solvation (MM-GBSA) module (Genheden and Ryde, 2015). MM-GBSA was used to estimate relative binding affinity for an FDA approved drugs against the binding pocket of $\mathrm{M}^{\text {pro }}$. We shortlisted 50 potential drugs from FDA approved library based upon both XP docking energy and MM-GBSA scores (Table 1). Molecular docking of $>2200$ FDA approved drugs at the similar hydrophobic core resulted in more than 50 potential drug molecules having docking score of $<5.0$ and MM-GBSA score of <30 (Table 1). The docking and MM-GBSA scores suggests that these drugs may have favorable interactions and hence may potentially inhibit $\mathrm{M}^{\text {pro }}$ activity. The best docking pose and the ligand protein interactions for the top nine hits have been shown in Figures 3-5.

The lowest docking score and MM-GBSA based binding energy of $-9.93 \mathrm{Kcal} / \mathrm{mol}$ and $77.27 \mathrm{Kcal} / \mathrm{mol}$, respectively were observed for iopamidol. Iopamidol interacts with the substrate binding site by forming hydrogen bonds with His164, Glu166, Gln189 and Thr190 and several non-bonded interactions. Iopamidol is a radiopaque contrast agent that contains iodine, and is used for imaging of blood vessels, organs, and other non-bony tissues on a CT scan or other radiologic (X-ray) examination (Bassi et al., 1982). Like iopamidol, another radiocontrast agent metrizamide was also among the top drugs in our list (Azuma et al., 1989; Ekholm et al., 1983). Another top 
molecule in our list was mitoxantrone having docking score of $-8.3 \mathrm{Kcal} / \mathrm{mol}$ and MM-GBSA score of $-69.9 \mathrm{Kcal} / \mathrm{mol}$. Binding of mitoxantrone is mediated by several non-bonded interactions and a network of hydrogen bonds with Glu166, Thr190, and Gln189. Mitoxantrone is an anticancer agent and immune suppressor agent used for treating cancer and multiple sclerosis (Hajihassan and Rabbani-Chadegani, 2009; Mazerski et al., 1998; Wu et al., 2013). Interestingly, this drug has also been reported as a potential $\mathrm{M}^{\text {pro }}$ binder by Ayman et al (Ayman et al., 2020).

Lumefantrine with docking score of $-5.136 \mathrm{Kcal} / \mathrm{mol}$ and the MM-GBSA score of -66.8 $\mathrm{Kcal} / \mathrm{mol}$ is at the third position in our list. It binds $\mathrm{M}^{\mathrm{pro}}$ with several non-bonded interactions, and forms hydrogen bond with Glu166 and $\pi-\pi$ interaction with His41. Lumefantrine is an antimalarial agent used in treating acute uncomplicated malaria (Kloprogge et al., 2018). Next drug in our list is dipyridamole which is phosphodiesterase 2 inhibitor that blocks the metabolism and uptake of adenosine by erythrocytes and vascular endothelial cells (Diener et al., 1996). It causes blood vessel dilation and inhibits blood clot formation and is used as coronary vasodilator (Diener et al., 1996). Docking analysis suggested that dipyridamole forms both hydrophobic and hydrophilic interactions with $\mathrm{M}^{\text {pro }}$ and interacts with Asn142 and Leu141 through hydrogen bonding. Acebutolol is another potential drug that showed favorable binding in our docking studies having docking score of $-7.3 \mathrm{Kcal} / \mathrm{mol}$ and MM-GBSA score of $-61.42 \mathrm{Kcal} / \mathrm{mol}$. Docking analysis suggested that acebutolol is stabilized by hydrophobic interactions and hydrogen bonding with Asn142, His164 and Gln189 residues in the $\mathrm{M}^{\text {pro }}$ binding pocket. Acebutolol is a cardio-selective, $\beta$-adrenoreceptor blocking agent. Acebutolol, is used for treating high blood pressure and it reduces the irregular heartbeat (Singh et al., 1986). Ospemifene is used for the treatment of dyspareunia and has non-hormonal estrogen receptor modulating activity (Rutanen et al., 2003; Wurz et al., 2014). This drug also forms hydrophobic interactions and hydrogen bonding and interacts with Thr190 in the binding pocket. The next drug in this list is dihydroergotamine, a derivative of ergotamine and mainly used for the treatment of acute migraine. It docks with binding score of $-6.3 \mathrm{Kcal} / \mathrm{mol}$ and MM-GBSA score $-60.62 \mathrm{Kcal} / \mathrm{mol}$. Besides hydrophobic interactions, it forms hydrogen bonds with Thr190, His 41 and Asn142. Dihydroergotamine can be administered as nasal spray; therefore, it can potentially be effective against pulmonary indications of COVID19 infection (Shrewsbury et al., 2008). Neratinib and palbociclib are two other anticancer drugs that appeared in our list which are used to treat early stage HER2- positive or HER2-negative breast cancer patients, respectively (Cadoo et al., 2014; Pernas and Tolaney, 2019). 
Hexoprenaline, which functions as bronchodilator, antiasthmatic, and tocolytic agent by stimulating $\beta-2$ adrenergic receptors, is among the top 10 compounds in the list (Pinder et al., 1977). Besides several non-bonded interactions, hexoprenaline forms hydrogen bonds with Phe140, Glu166 and Thr190. Riboflavin (Vitamin B2) is at fifteenth position in our list. It binds with docking and MM-GBSA scores of $-7.29 \mathrm{Kcal} / \mathrm{mol}$ and $-53.66 \mathrm{Kcal} / \mathrm{mol}$, respectively. It forms a hydrogen bond with Leu141, Gly143 and Glu166 besides having several non-bonded interactions. Pantethine, (vitamin B5) also appeared at the $31^{\text {st }}$ position in the list. Both riboflavin and pantethine, though having relatively lower ranks based on docking and MM-GBSA scores, being the safest biomolecules among the list can be taken forward to evaluate $\mathbf{M}^{\text {pro }}$ inhibitory activities. Bioavailability, cell penetration, half-life and safety profile make riboflavin and pantethine potential candidates to be screened as an inhibitor of $\mathrm{M}^{\text {pro }}$.

\section{Molecular Dynamics (MD) simulations}

In order to understand the dynamics of $\mathrm{M}^{\text {pro }}$-drug interactions, we selected $\mathrm{M}^{\text {pro-lumefantrine and }}$

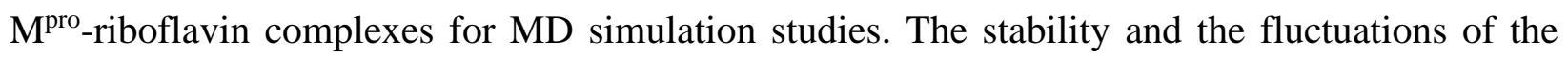
$\mathrm{M}^{\text {pro }}$-drug complex structures were studied by examining the RMSD and the RMSF plots of the protein $\mathrm{C} \alpha$ and ligand. The convergence of RMSD trajectory of $\mathrm{M}^{\text {pro }}$ - drug complexes during 20$30 \mathrm{~ns}$ MD simulations suggests that the complexes are stable, and the ligand was bound favorably to the substrate binding pocket. The low RMSD observed for the protein backbone suggests that the protein is stable and do not undergo overall major conformational changes during the simulation run (Figure 6A and 6B). 


\section{Materials and Methods}

\section{Multiple sequence alignment and ConSurf analysis}

For multiple sequence alignment, $\mathrm{M}^{\text {pro }}$ sequences of SARS-CoV-2, SARS-CoV, MERS, human coronavirus NL63 (H-CoV), infectious bronchitis virus (IBV), porcine epidemic diarrhea virus (PEDV) and bat coronavirus were extracted from NCBI database (www.ncbi.nlm.nih.gov) and then aligned using COBALT tool (Papadopoulos and Agarwala, 2007) using default settings. Then, multiple sequence alignment file was submitted to ESPRIPT 3.0 server (Robert and Gouet, 2014) for rendering sequence similarity and secondary structure information from the aligned sequences. The PDB ID: 6M03, SARC-Cov-2 M $\mathrm{M}^{\text {ro }}$, was used for secondary structure assignment. ConSurf analysis was performed to generate conservation score on PDB ID: 6M03 using HMMER homology search algorithm with E-value 0.0001 and UNIREF-90 protein database and multiple sequence alignment was generated using MAFFT. Fifty sequences sharing $>60 \%$ sequence identity was used for ConSurf analysis (Ashkenazy et al., 2010).

\section{FDA approved small molecule library preparation}

The FDA approved drug database was downloaded from drug bank database (www.drugbank.ca) and prepared for docking study using the LigPrep module in Schrodinger software suite to create energy minimized 3D molecular structures (Chen and Foloppe, 2010). Downloaded FDA drug compound databases contain only 2D molecular structures in SDF format. LigPrep efficiently and accurately performed 3D conversion of these FDA approved drug compounds. Now, this 3D structure compound library can utilize for docking studies.

\section{Structure-based in-silico screening and scoring}

The crystal structure of $\mathrm{M}^{\text {pro }}$ solved in complex with an inhibitor N3, PDB ID: 6LU7 was used as a receptor (Jin et al., 2020). The structure was prepared using the protein preparation wizard module using Maestro interface of the Schrodinger software suite. The prepared structure was used to identify potential binding sites using the SiteMap module, which uses site score function to rank the possible binding sites according to size, functionality, and extent of solvent exposure on the protein (Halgren, 2007; Halgren, 2009). The site with a site score of $>0.9$ was used for grid 
generation using the Receptor Grid Generation module. The molecules were docking using Xtraprecision (XP) mode in Glide module (Friesner et al., 2006).

\section{Binding energies calculation using MM-GBSA tool}

The binding energies of $\mathrm{M}^{\text {pro }}$ with docked FDA drugs were calculated using the MM-GBSA module in the Schrödinger software suite (Genheden and Ryde, 2015). The docked protein and ligand complex were separated manually and loaded as receptor or ligand, respectively in the MMGBSA module. MM-GBSA analysis includes five types of different energy calculation methods including optimization of free receptor, optimization free ligand, optimization complex, receptor from minimized complex, ligand from the minimized complex (Genheden and Ryde, 2015).

\section{Molecular Dynamics Simulations}

The docked protein-drug were subjected to system builder panel of the Desmond module incorporated in Schrödinger software suite (Bowers et al., 2006). The complexes were embedded in an orthorhombic box with the buffer distance of a, b, c =10 А. The system was solvated by adding water molecules (TIP-3P solvation system) and $\mathrm{NaCl}$ concentration was kept at $150 \mathrm{mM}$. The model system was relaxed using multi-step Desmond relaxation protocol. The simulation was carried out at $300 \mathrm{~K}$ and 1.013 bar for $20 \mathrm{~ns}$ with trajectory recording intervals of 20 ps and energy recording intervals of $1.2 \mathrm{ps}$ in NPT ensemble class. OPLS3 force field was used for MD simulations (Harder et al., 2016). 


\section{Discussion}

Recent COVID-19 outbreak caused by the beta-coronavirus, SARS-CoV-2, has caused immense human and economic losses fueling efforts to discover effective cure and therapeutics. In search for such potential therapeutic agents, we used in silico structure-based drug design and drug repurposing strategies. Several groups have targeted $\mathbf{M}^{\text {pro }}$ for developing effect drugs against SARS-CoV-2. Yang et al, designed an effective inhibitor N3 which binds at the substrate binding site and inactivates SARS-CoV (Yang et al., 2005). Recently, using computer-aided drug design strategy, Jin et al, too reported N3 to be mechanism-based inhibitor of SARS-CoV-2 (Jin et al., 2020). Khaerunnisa et al, implemented repurposing strategies to find out possible potential inhibitors against $\mathrm{M}^{\text {pro }}$. They reported nelfinavir and lopinavir to be potential inhibitors of $\mathrm{M}^{\text {pro }}$ (Khaerunnisa and Soetjipto, 2020). In another study, based on docking studies, Adem et al., found hesperidin, rutin, fiosmin and other natural polyphenols to be more effective against $\mathrm{M}^{\text {pro }}$ than nelfinavir (Adem, 2020). Jin et al., reported Ebselen to exhibit excellent inhibitory activity against $\mathrm{M}^{\mathrm{pro}}$ with an $\mathrm{IC}_{50} 0.67 \mu \mathrm{M}$ (Jin et al., 2020).

In our study, two radio contrast agents were among the top hits. However, these drugs have multiple side effects varying in severity from itching to contrast-induced nephropathy- a lifethreatening emergency (Andreucci et al., 2014). Therefore, these may not be suitable candidates for treating COVID-19. Similarly, there are two anticancer drugs (mitoxantrone and neratinib) in the top hits which may have undesirable side effects, hence, may not be suitable. Lumefantrine, one of the top drugs in our list, has limited side effects and longer half-life (three to six days) which makes it a potential drug that may be taken up for further studies to test efficacy in inhibiting $\mathrm{M}^{\text {pro }}$ and hence in treating COVID-19. Chloroquine, another antimalarial drug in clinical use, has already been established to have potential in treating COVID-19, however, it uses distinct mechanism of action. Similarly, riboflavin is among top drugs which is a safe and shows good potential binding to $\mathrm{M}^{\text {pro }}$. Besides these dipyridamole, dihydroergotamine and hexoprenaline are other drugs in top list and have good bioavailability and less toxicity. Dihydroergotamine has an added advantage over other drugs as it can be administered as a nasal spray, hence can readily target the site of infection i.e. lungs. Using molecular docking studies, Narayanan et al., recently reported vitamin B12 to be a potential inhibitor of RNA-dependent-RNA polymerase (Narayanan, 
2020). So, these vitamins B2, B5, and B12 combination may also be explored as potential treatment for COVID-19 that may exploit distinct mechanisms for inhibiting viral replication.

To conclude, among several hits obtained in our molecular docking studies, lumefantrine, dihydroergotamine, hexoprenaline, riboflavin and pantethine appeared attractive candidates as potential inhibitors of $\mathrm{M}^{\text {pro }}$. However, in vitro and in vivo studies are necessary to further investigate their therapeutic potential in treating COVID-19 or other related coronavirus infections.

\section{Acknowledgments}

KGT would like to acknowledge members of Structural Biology Laboratory, CSIR-IMTECH for useful suggestions and discussions. This work was supported by grants to KGT by Council of Scientific and Industrial Research (CSIR), India. DV, SK, and SD are CSIR-SRF, DBT-SRF and CSIR-Nehru PDF, respectively.

\section{Conflict of interest}

The authors declare that there is no conflict of interest. 


\section{Reference}

Adem, S.E., V.; Sarfraz, I.; Rasul, A.; Ali, M (2020). Identification of Potent COVID-19 Main Protease (Mpro) Inhibitors from Natural Polyphenols: An in Silico Strategy Unveils a Hope against CORONA.

Al-Tawfiq, J.A., Al-Homoud, A.H., and Memish, Z.A. (2020). Remdesivir as a possible therapeutic option for the COVID-19. Travel Med Infect Dis, 101615.

Andreucci, M., Solomon, R., and Tasanarong, A. (2014). Side effects of radiographic contrast media: pathogenesis, risk factors, and prevention. Biomed Res Int 2014, 741018.

Arowosegbe, M.A., Amusan, O.T., Adeola, S.A., Adu, O.B., Akinola, I.A., Ogungbe, B.F., Omotuyi, O.I., Saibu, G.M., Ogunleye, A.J., Kanmodi, R.I., et al. (2019). Kaempferol as a Potential PAK4 Inhibitor in Triple Negative Breast Cancer: Extra Precision Glide Docking and Free Energy Calculation. Curr Drug Discov Technol.

Ashkenazy, H., Erez, E., Martz, E., Pupko, T., and Ben-Tal, N. (2010). ConSurf 2010: calculating evolutionary conservation in sequence and structure of proteins and nucleic acids. Nucleic Acids Res 38, W529-533.

Ayman, F., Ping, W., Mahmoud, A., and Hesham, S. (2020). Identification of FDA Approved Drugs Targeting COVID-19 Virus by Structure-Based Drug Repositioning.

Azuma, H., Nomura, S., Ikoma, Y., Yokoyama, M., and Oshino, N. (1989). A possible mechanism for the neural adverse reactions caused by metrizamide. Fortschr Geb Rontgenstrahlen Nuklearmed Erganzungsbd 128, 134-142.

Bassi, P., Cecchini, A., Dettori, P., and Signorini, E. (1982). Myelography with iopamidol, a nonionic water- soluble contrast medium: incidence of complications. Neuroradiology 24, 85-90.

Bowers, K., Chow, E., Huageng, X., Dror, R.O., Eastwood, M.P., Gregersen, B., Klepeis, J., Kolossváry, I., Moraes, M.A., Sacerdoti, F.D., et al. (2006). Scalable Algorithms for Molecular Dynamics Simulations on Commodity Clusters.

Brierley, I., Boursnell, M.E., Binns, M.M., Bilimoria, B., Blok, V.C., Brown, T.D., and Inglis, S.C. (1987). An efficient ribosomal frame-shifting signal in the polymerase-encoding region of the coronavirus IBV. EMBO J 6, 3779-3785. 
Cadoo, K.A., Gucalp, A., and Traina, T.A. (2014). Palbociclib: an evidence-based review of its potential in the treatment of breast cancer. Breast Cancer (Dove Med Press) 6, 123-133.

Chen, I.J., and Foloppe, N. (2010). Drug-like bioactive structures and conformational coverage with the LigPrep/ConfGen suite: comparison to programs MOE and catalyst. J Chem Inf Model $50,822-839$.

Diener, H.C., Cunha, L., Forbes, C., Sivenius, J., Smets, P., and Lowenthal, A. (1996). European Stroke Prevention Study. 2. Dipyridamole and acetylsalicylic acid in the secondary prevention of stroke. J Neurol Sci 143, 1-13.

Ekholm, S.E., Reece, K., Coleman, J.R., Kido, D.K., and Fischer, H.W. (1983). Metrizamide--a potential in vivo inhibitor of glucose metabolism. Radiology 147, 119-121.

Friesner, R.A., Murphy, R.B., Repasky, M.P., Frye, L.L., Greenwood, J.R., Halgren, T.A., Sanschagrin, P.C., and Mainz, D.T. (2006). Extra precision glide: docking and scoring incorporating a model of hydrophobic enclosure for protein-ligand complexes. J Med Chem 49, 6177-6196.

Gao, Y., Yan, L., Huang, Y., Liu, F., Zhao, Y., Cao, L., Wang, T., Sun, Q., Ming, Z., Zhang, L., et al. (2020). Structure of RNA-dependent RNA polymerase from 2019-nCoV, a major antiviral drug target. bioRxiv, 2020.2003.2016.993386.

Ge, Y., Tian, T., Huang, S., Wan, F., Li, J., Li, S., Yang, H., Hong, L., Wu, N., Yuan, E., et al. (2020). A data- driven drug repositioning framework discovered a potential therapeutic agent targeting COVID-19. bioRxiv, 2020.2003.2011.986836.

Genheden, S., and Ryde, U. (2015). The MM/PBSA and MM/GBSA methods to estimate ligandbinding affinities. Expert Opin Drug Discov 10, 449-461.

Guo, Y.-R., Cao, Q.-D., Hong, Z.-S., Tan, Y.-Y., Chen, S.-D., Jin, H.-J., Tan, K.-S., Wang, D.Y., and Yan, Y.

(2020). The origin, transmission and clinical therapies on coronavirus disease 2019 (COVID-19) outbreak- an update on the status. Military Medical Research 7, 11. 
Hajihassan, Z., and Rabbani-Chadegani, A. (2009). Studies on the binding affinity of anticancer drug mitoxantrone to chromatin, DNA and histone proteins. J Biomed Sci 16, 31.

Halgren, T. (2007). New method for fast and accurate binding-site identification and analysis. Chem Biol Drug Des 69, 146-148.

Halgren, T.A. (2009). Identifying and characterizing binding sites and assessing druggability. J Chem Inf Model 49, 377-389.

Halgren, T.A., Murphy, R.B., Friesner, R.A., Beard, H.S., Frye, L.L., Pollard, W.T., and Banks, J.L. (2004). Glide: a new approach for rapid, accurate docking and scoring. 2. Enrichment factors in database screening. J Med Chem 47, 1750-1759.

Harder, E., Damm, W., Maple, J., Wu, C., Reboul, M., Xiang, J.Y., Wang, L., Lupyan, D., Dahlgren, M.K., Knight, J.L., et al. (2016). OPLS3: A Force Field Providing Broad Coverage of Drug-like Small Molecules and Proteins. J Chem Theory Comput 12, 281-296.

Hoffmann, M., Kleine-Weber, H., Schroeder, S., Kruger, N., Herrler, T., Erichsen, S., Schiergens, T.S., Herrler, G., Wu, N.H., Nitsche, A., et al. (2020). SARS-CoV-2 Cell Entry Depends on ACE2 and TMPRSS2 and Is Blocked by a Clinically Proven Protease Inhibitor. Cell https://doi.org/10.1016/j.cell.2020.02.052.

Jin, Z., Du, X., Xu, Y., Deng, Y., Liu, M., Zhao, Y., Zhang, B., Li, X., Zhang, L., Peng, C., et al. (2020). Structure of $M<$ sup>pro</sup> from COVID-19 virus and discovery of its inhibitors. bioRxiv, 2020.2002.2026.964882.

Khaerunnisa, S.K., H.; Awaluddin, R.; Suhartati, S.; , and Soetjipto, S. (2020). Potential Inhibitor of COVID- 19 Main Protease (Mpro) From Several Medicinal Plant Compounds by Molecular Docking Study. Preprints.

Kloprogge, F., Workman, L., Borrmann, S., Tekete, M., Lefevre, G., Hamed, K., Piola, P., Ursing, J., Kofoed, P.E., Martensson, A., et al. (2018). Artemether-lumefantrine dosing for malaria treatment in young children and pregnant women: A pharmacokinetic-pharmacodynamic meta-analysis. PLoS Med 15, e1002579.

Koonpaew, S., Teeravechyan, S., Frantz, P.N., Chailangkarn, T., and Jongkaewwattana, A. (2019). PEDV and PDCoV Pathogenesis: The Interplay Between Host Innate Immune Responses and Porcine Enteric Coronaviruses. Front Vet Sci 6, 34. 
Li, F. (2016). Structure, Function, and Evolution of Coronavirus Spike Proteins. Annu Rev Virol 3, 237-261. Mackenzie, J.S., and Smith, D.W. (2020). COVID-19: a novel zoonotic disease caused by a coronavirus from China: what we know and what we don't. Microbiol Aust, MA20013.

Mazerski, J., Martelli, S., and Borowski, E. (1998). The geometry of intercalation complex of antitumor mitoxantrone and ametantrone with DNA: molecular dynamics simulations. Acta Biochim Pol 45, 1-11.

McChesney, E.W. (1983). Animal toxicity and pharmacokinetics of hydroxychloroquine sulfate. Am J Med 75, 11-18.

Millet, J.K., and Whittaker, G.R. (2015). Host cell proteases: Critical determinants of coronavirus tropism and pathogenesis. Virus Res 202, 120-134.

Mott, D.J. (1988). A slice width and focal spot alignment camera for computed tomographic scanners. Br J Radiol 61, 1175-1176.

Narayanan, N.N., D.T (2020). Vitamin B12 May Inhibit RNA-Dependent-RNA Polymerase Activity of nsp12 from the SARS-CoV-2 Virus (Preprints).

Papadopoulos, J.S., and Agarwala, R. (2007). COBALT: constraint-based alignment tool for multiple protein sequences. Bioinformatics 23, 1073-1079.

Pernas, S., and Tolaney, S.M. (2019). HER2-positive breast cancer: new therapeutic frontiers and overcoming resistance. Ther Adv Med Oncol 11, 1758835919833519.

Pinder, R.M., Brogden, R.N., Speight, T.M., and Avery, G.S. (1977). Hexoprenaline: a review of its pharmacological properties and therapeutic efficacy with particular reference to asthma. Drugs 14, 1-28. Prajapat, M., Sarma, P., Shekhar, N., Avti, P., Sinha, S., Kaur, H., Kumar, S., Bhattacharyya, A., Kumar, H., Bansal, S., et al. (2020). Drug targets for corona virus: A systematic review. Indian J Pharmacol 52, 56-65. Robert, X., and Gouet, P. (2014). Deciphering key features in protein structures with the new ENDscript server. Nucleic Acids Res 42, W320324.

Rutanen, E.M., Heikkinen, J., Halonen, K., Komi, J., Lammintausta, R., and Ylikorkala, O. (2003). Effects of ospemifene, a novel SERM, on hormones, genital tract, climacteric symptoms, and quality of life in postmenopausal women: a double-blind, randomized trial. Menopause 10, 433-439. 
Schoeman, D., and Fielding, B.C. (2019). Coronavirus envelope protein: current knowledge. Virology Journal 16, 69.

Shrewsbury, S.B., Cook, R.O., Taylor, G., Edwards, C., and Ramadan, N.M. (2008). Safety and pharmacokinetics of dihydroergotamine mesylate administered via a Novel (Tempo) inhaler. Headache 48, 355-367.

Simmons, G., Gosalia, D.N., Rennekamp, A.J., Reeves, J.D., Diamond, S.L., and Bates, P. (2005). Inhibitors of cathepsin L prevent severe acute respiratory syndrome coronavirus entry. Proc Natl Acad Sci U S A 102, 11876-11881.

Singh, B.N., Thoden, W.R., and Wahl, J. (1986). Acebutolol: a review of its pharmacology, pharmacokinetics, clinical uses, and adverse effects. Pharmacotherapy 6, 45-63. 
Walls, A.C., Tortorici, M.A., Snijder, J., Xiong, X., Bosch, B.J., Rey, F.A., and Veesler, D. (2017). Tectonic conformational changes of a coronavirus spike glycoprotein promote membrane fusion. Proc Natl Acad Sci U S A 114, 11157-11162.

Wu, C.C., Li, Y.C., Wang, Y.R., Li, T.K., and Chan, N.L. (2013). On the structural basis and design guidelines for type II topoisomerase-targeting anticancer drugs. Nucleic Acids Res 41, 10630-10640.

Wurz, G.T., Kao, C.J., and DeGregorio, M.W. (2014). Safety and efficacy of ospemifene for the treatment of dyspareunia associated with vulvar and vaginal atrophy due to menopause. Clin Interv Aging 9, 1939- 1950.

Xue, X., Yu, H., Yang, H., Xue, F., Wu, Z., Shen, W., Li, J., Zhou, Z., Ding, Y., Zhao, Q., et al. (2008). Structures of two coronavirus main proteases: implications for substrate binding and antiviral drug design. J Virol 82, 2515-2527.

Yang, H., Xie, W., Xue, X., Yang, K., Ma, J., Liang, W., Zhao, Q., Zhou, Z., Pei, D., Ziebuhr, J., et al. (2005). Design of wide-spectrum inhibitors targeting coronavirus main proteases. PLoS Biol 3, e324. 
Table 1: List of top 51 hits with their respective docking and MM-GBSA scores and current clinical use of the drugs.

\begin{tabular}{|c|c|c|c|c|}
\hline S. No. & Drug & $\begin{array}{l}\text { Docking } \\
\text { score }\end{array}$ & $\begin{array}{l}\text { MM-GBSA } \\
\text { score }\end{array}$ & Clinical use \\
\hline 1 & Iopamidol & -9.93 & -72.77 & Radiocontrast agent \\
\hline 2 & Mitoxantrone & -8.354 & -69.9 & Anti-cancer \\
\hline 3 & Lumefantrine & -5.136 & -66.8 & Antimalarial \\
\hline 4 & Dipyridamole & -7.193 & -65.56 & Vasodilator \\
\hline 5 & Acebutolol & -7.397 & -61.42 & Anti-arrhythmia \\
\hline 6 & Ospemifene & -6.58 & -60.68 & Estrogen receptor modulator \\
\hline 7 & Dihydroergotamine & -6.301 & -60.62 & Vasoconstrictor \\
\hline 8 & Neratinib & -6.564 & -59.57 & Anti-cancer \\
\hline 9 & Palbociclib & -6.297 & -59.51 & Anti-cancer \\
\hline 10 & Hexoprenaline & -6.352 & -58.35 & Anti-asthmatic \\
\hline 11 & Prazepam & -6.28 & -57.77 & Anxiolytic, sedative \\
\hline 12 & Dipivefrin & -6.254 & -57.56 & Anti-glaucoma \\
\hline 13 & Doxorubicin & -6.217 & -56.45 & Anti-cancer \\
\hline 14 & Rosuvastatin & -6.443 & -53.93 & Anti-obesity \\
\hline 15 & Riboflavin & -7.219 & -53.66 & Vitamin B2 \\
\hline 16 & Iopromide & -7.392 & -51.78 & Radiocontrast agent \\
\hline 17 & Afatinib & -6.822 & -49.6 & Anti-cancer \\
\hline 18 & Fluvastatin & -6.628 & -49.45 & Anti-obesity \\
\hline 19 & Metrizamide & -8.449 & -49.24 & Radiocontrast agent \\
\hline 20 & Pitavastatin & -6.468 & -49.17 & Anti-obesity \\
\hline 21 & Talniflumate & -6.763 & -48.31 & Anti-inflammatory \\
\hline 22 & Lucanthone & -6.741 & -47.93 & Schistosomicides \\
\hline 23 & Prezatide & -7.183 & -47.55 & Wound healing \\
\hline 24 & Fluvoxamine & -6.348 & -46.46 & Antidepressant \\
\hline 25 & Canagliflozin & -6.469 & -46.44 & Ant-diabetic \\
\hline 26 & Xanthinol & -6.981 & -45.04 & Vasodilator \\
\hline 27 & Pravastatin & -6.283 & -44.31 & Anti-hypercholesterolemia \\
\hline 28 & Fominoben & -6.093 & -44.31 & Anti-tussive \\
\hline 29 & Esculin & -6.25 & -44.28 & Antioxidant \\
\hline 30 & Imipenem & -6.979 & -42.5 & Antibiotic \\
\hline 31 & Betaxolol & -6.625 & -41.1 & Anti-hypertension \\
\hline 32 & Pantethine & -6.365 & -40.82 & Vitamin B5 \\
\hline 33 & $\begin{array}{l}\text { Benzylpenicilloyl } \\
\text { polylysine }\end{array}$ & -6.438 & -40.41 & Anti-histamine \\
\hline 34 & Arbutin & -6.647 & -39.09 & Skin lightening, anti-melanin \\
\hline 35 & Iron saccharate & -7.018 & -38.87 & Treatment for iron deficiency anemia \\
\hline 36 & Methoxamine & -6.216 & -38.85 & Anti-acute hypotensive \\
\hline 37 & Primaquine & -6.398 & -38.73 & Antimalaria \\
\hline 38 & Lactulose & -8.564 & -37.12 & Laxative \\
\hline 39 & Midodrine & -6.057 & -33.77 & Vasoconstrictor, anti-hypotension \\
\hline 40 & Glutathione disulfide & -6.191 & -23.29 & Antioxidant \\
\hline 41 & Pravastatin & -6.283 & -44.31 & Anti-hypercholesterolemia \\
\hline 42 & Halofantrine & -5.972 & -59.62 & Antimalarial \\
\hline
\end{tabular}




\begin{tabular}{|l|l|c|c|l|}
\hline 43 & Streptomycin & -5.582 & -59.12 & Antibiotic \\
\hline 44 & Vandetanib & -5.19 & -57.33 & Anti-cancer \\
\hline 45 & Ditazole & -5.064 & -57.24 & Anti-inflammatory \\
\hline 46 & Misoprostol & -5.935 & -55.93 & Prostaglandin analogue \\
\hline 47 & Zuclopenthixol & -5.788 & -55.03 & Antipsychotic \\
\hline 48 & Lenvatinib & -5.998 & -54.65 & Anti-cancer \\
\hline 49 & Olmutinib & -5.205 & -54.62 & Anti-cancer \\
\hline 50 & Adefovir Dipivoxil & -5.085 & -53.62 & Antiviral \\
\hline 51 & Flavoxate & -5.129 & -53.26 & Anticholinergic \\
\hline
\end{tabular}




\section{Figures}
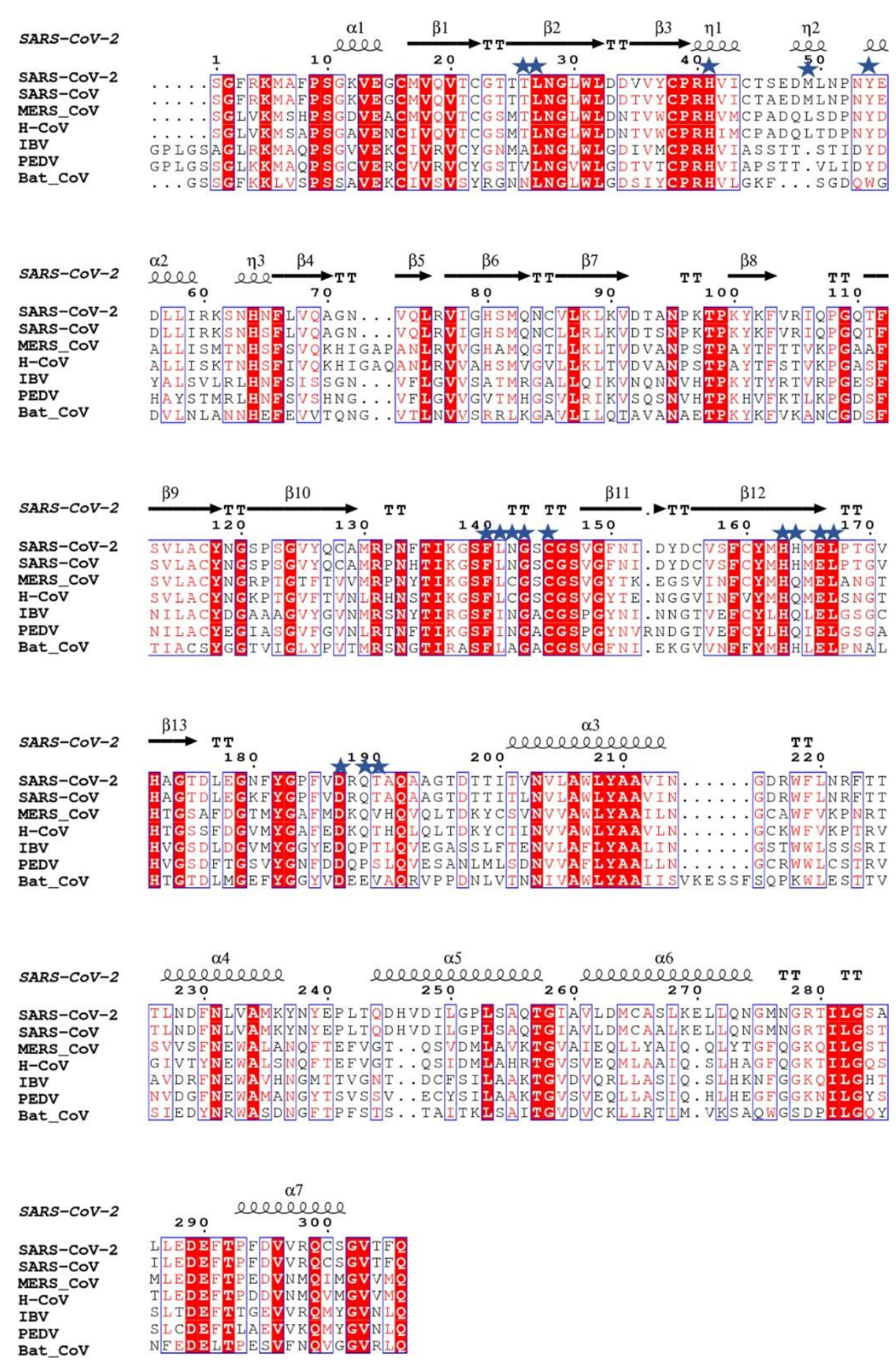

Figure 1. Multiple sequence alignment of $M^{\text {pro }}$ of SARS-CoV-2 with $M^{\text {pro }}$ of other coronaviruses performed using ESPRIPT 3.0 server. PDB ID: 6M03 was used as a reference for assignment of secondary structural elements. The conserved residues involved in interaction with ligands are highlited with blue stars. 

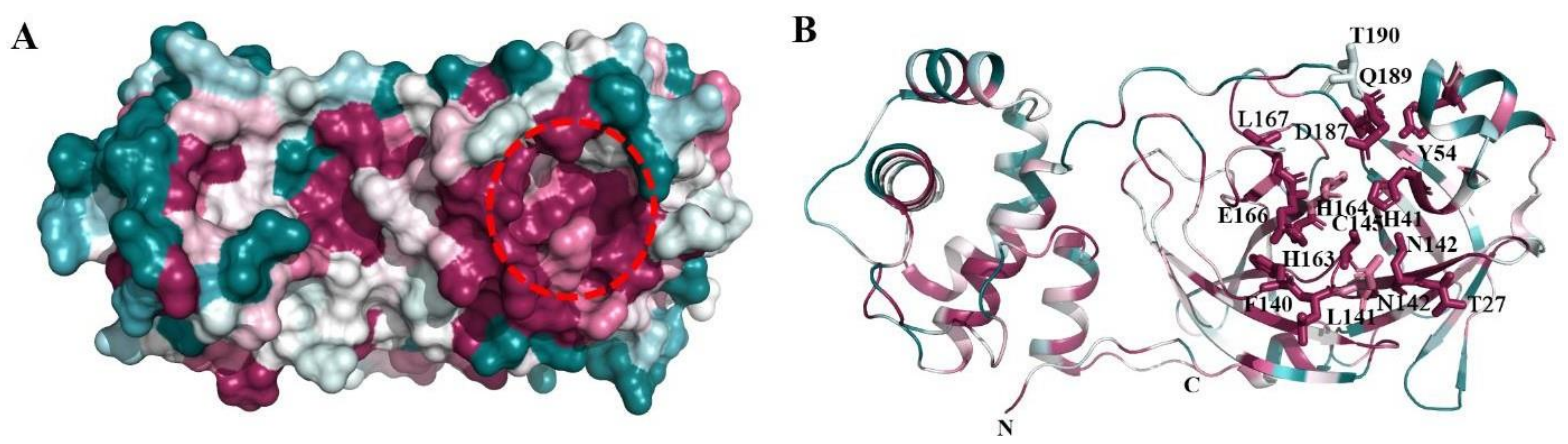

Figure 2. ConSurf analysis showing conserved regions in the three-dimensional structure of $\mathrm{M}^{\text {pro }}$.

(A) Surface view showing the highly conserved substrate binding site (red broken circle) and region involved in homodimeraization (broken blue circle). Drugs were docked in the substrate binding region of $\mathrm{M}^{\text {pro }}$. (B) Cartoon representation showing the conserved regions in $\mathrm{M}^{\text {pro }}$. The residues forming the substrate binding pocket are shown in stick representation. 
A

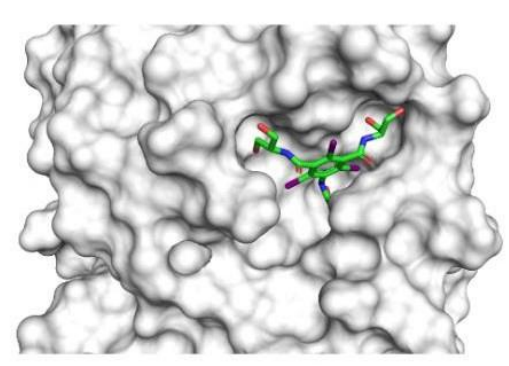

Iopamidol

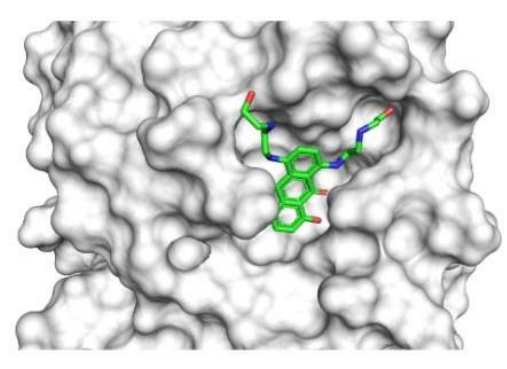

Mitoxantrone

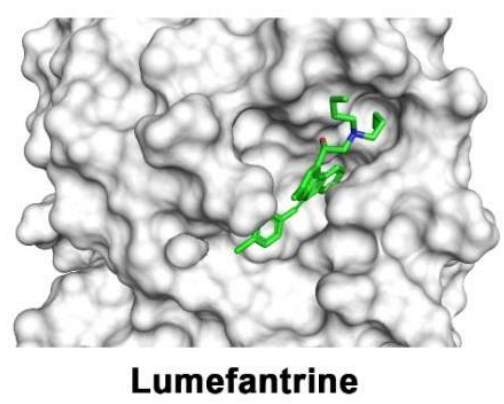

B

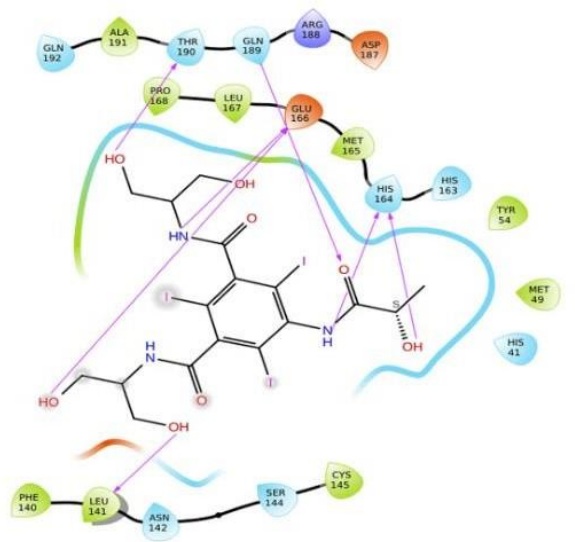

inin

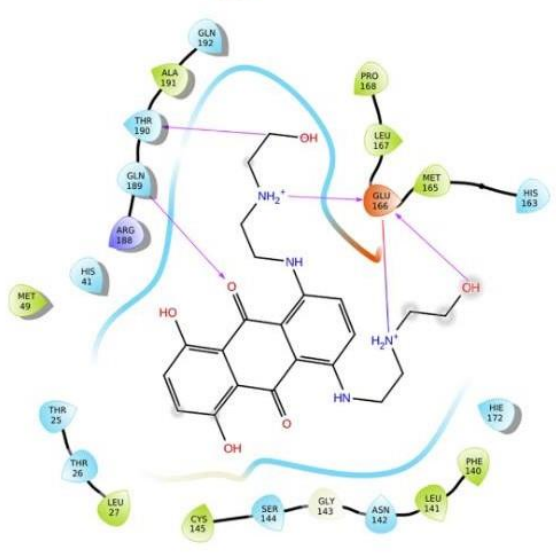

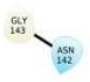

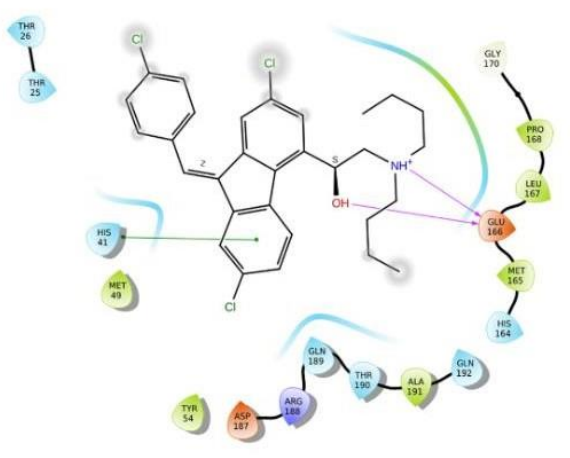

Charged
Hydrophobic $\rightarrow$ Polar
$\longmapsto$ Pi-Pi interaction

Figure 3: (A) Docking of FDA approved drugs (shown in stick representation) iopamidol, mitoxantrone, and lumefantrine in the substrate binding pocket of $\mathrm{M}^{\text {pro }}$ (Grey surface representation). Carbon, nitrogen and oxygen are shown in green, blue and red colours, respectively. (B) 2-D interaction map of drugs with $\mathrm{M}^{\text {pro }}$ highlighting various interactions stabilizing protein-drug interactions. 
A
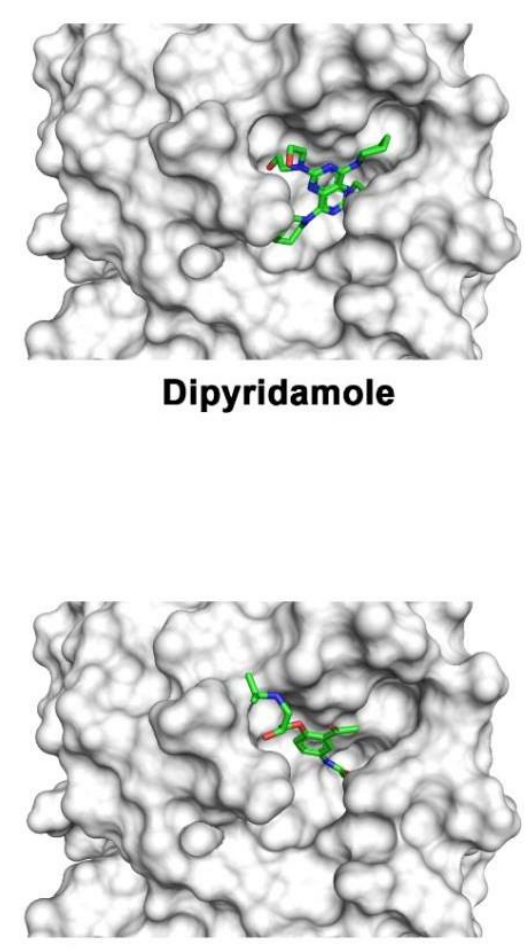

Acebutalol

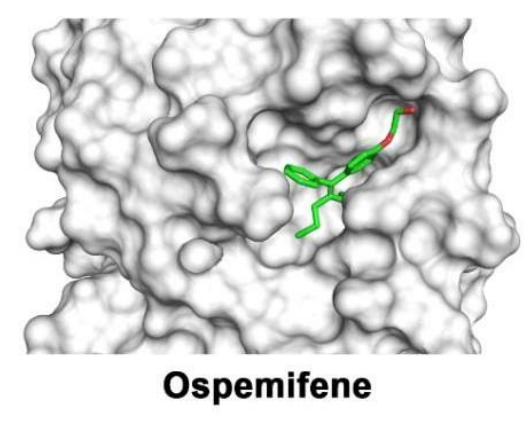

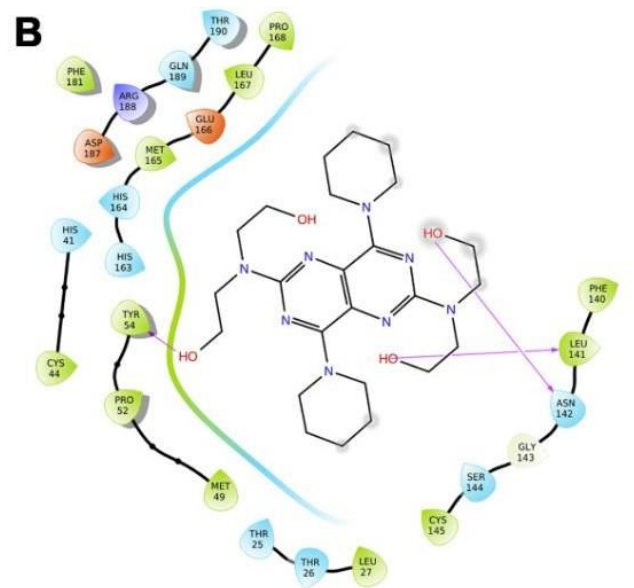
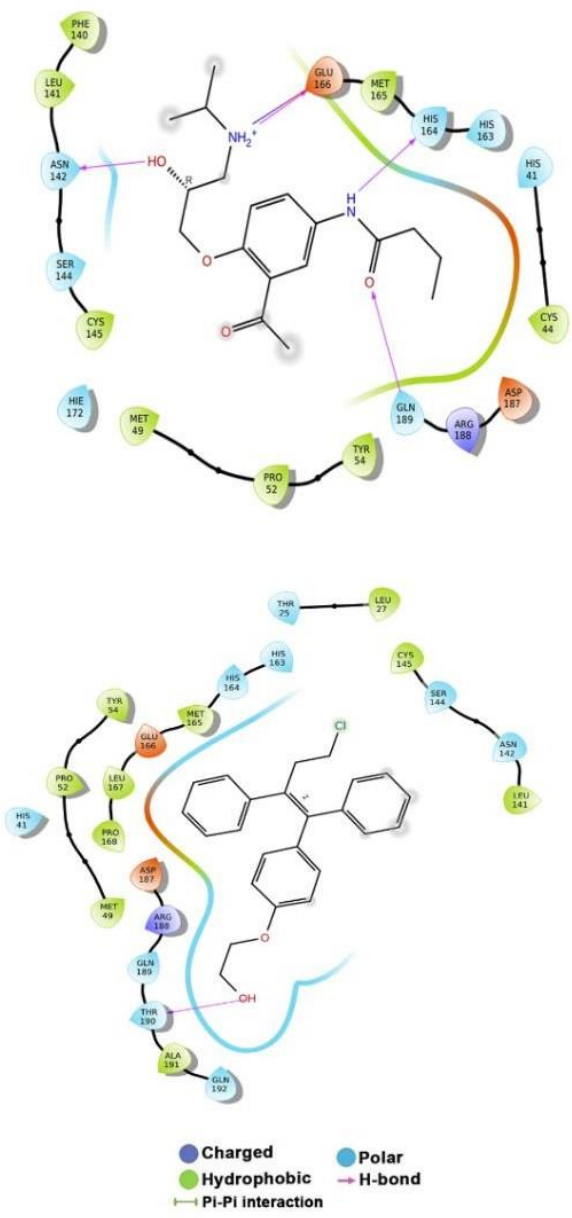

Figure 4: (A) Docking of FDA approved drugs (shown in stick representation) dipyridamole, acebutalol, and ospemifine in the substrate binding pocket of $\mathrm{M}^{\text {pro }}$ (Grey surface representation). Carbon, nitrogen and oxygen are shown in green, blue and red colours, respectively. (B) 2-D interaction map of drugs with $\mathrm{M}^{\text {pro }}$ highlighting various interactions stabilizing protein-drug interactions. 
A

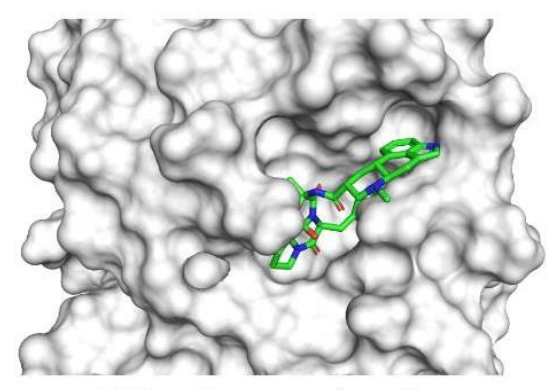

Dihydroergotamine
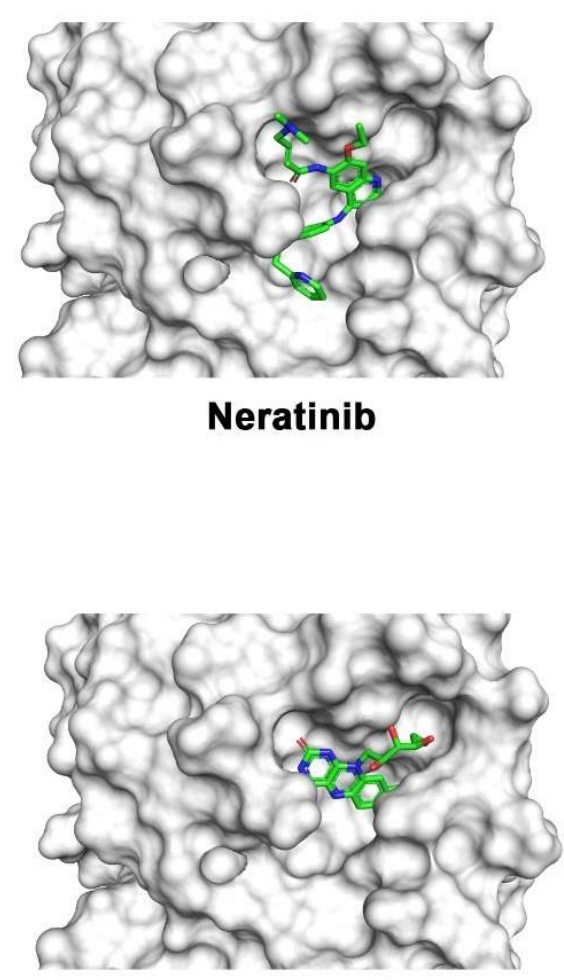

Riboflavin
B
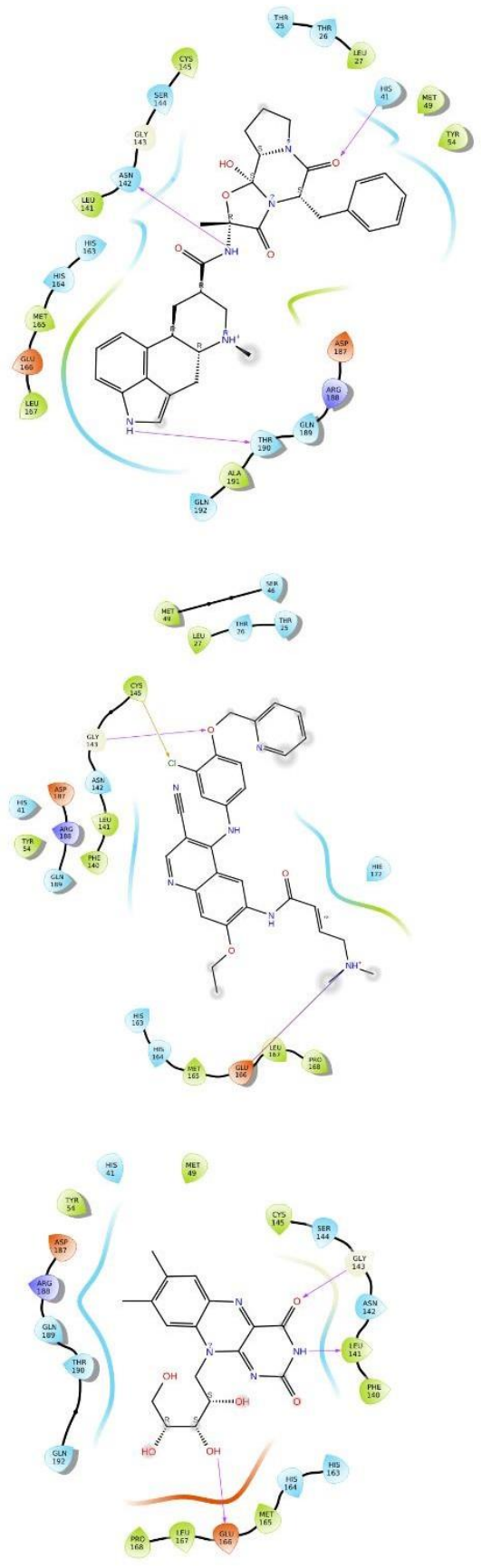

Charged
Hydrophobic $\rightarrow$ Polar
$\rightarrow$ Pi-Pi interaction

Figure 5: (A) Docking of FDA approved drugs (shown in stick representation) dihydroergotamine, neratinib, and riboflavin in the substrate binding pocket of $\mathrm{M}^{\text {pro }}$ (Grey surface representation). Carbon, nitrogen and oxygen are shown in green, blue and red colours, respectively. (B) 2-D interaction map of drugs with $\mathrm{M}^{\text {pro }}$ highlighting various interactions stabilizing protein-drug interactions. 
A

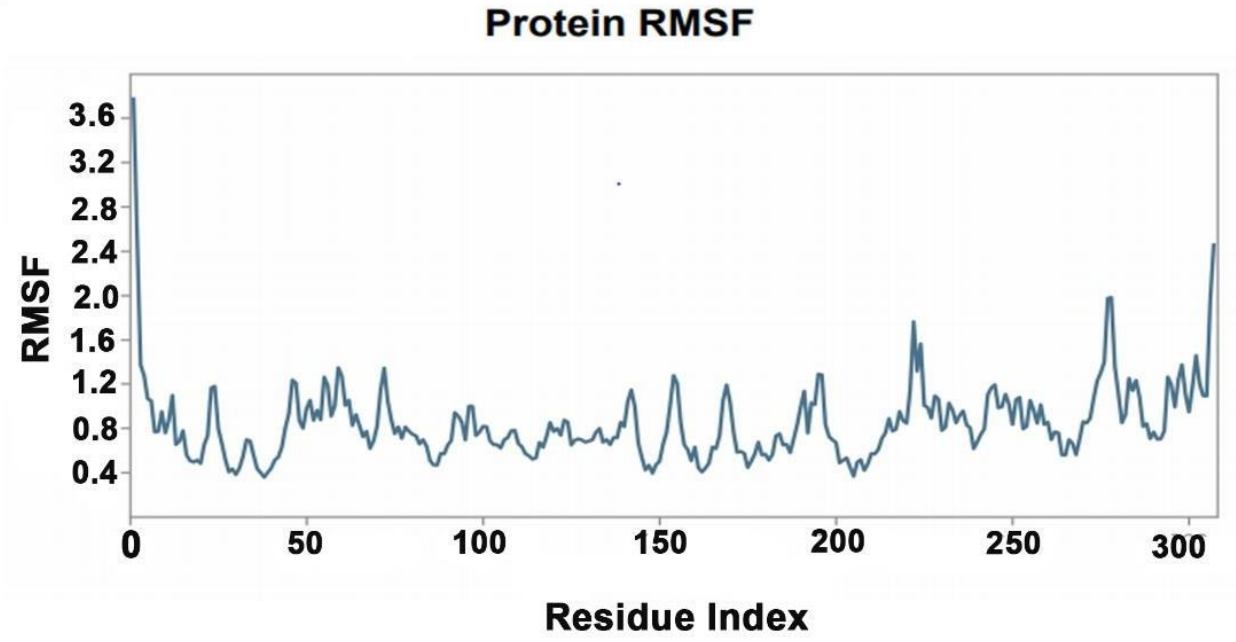

B

Protein-Ligand RMSD

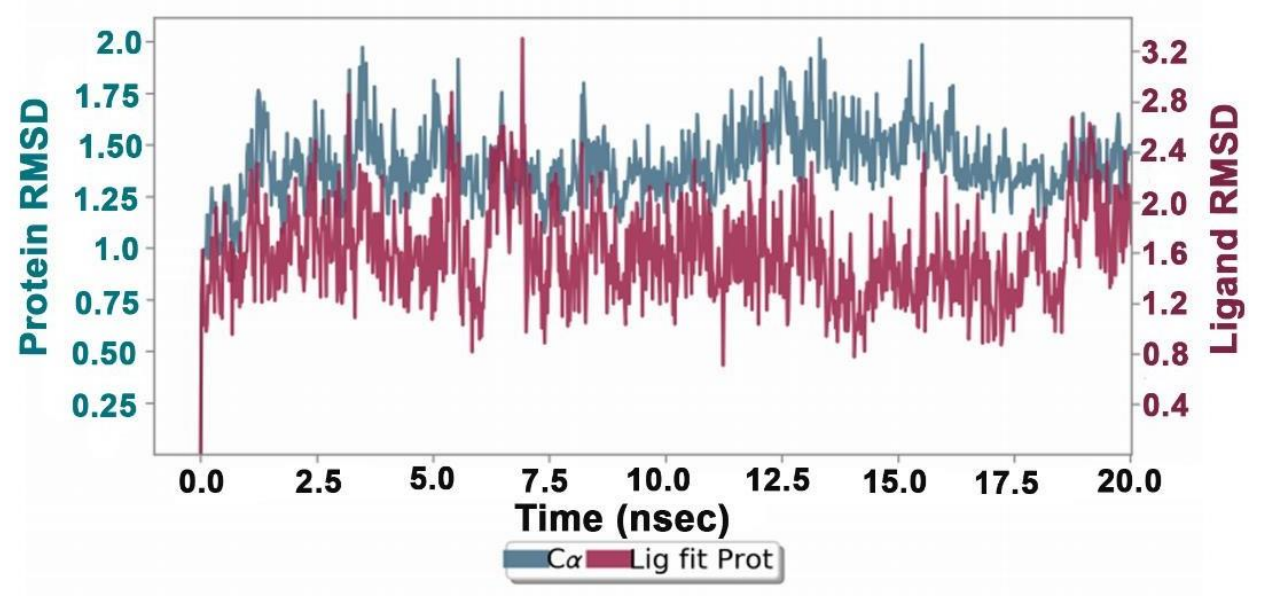

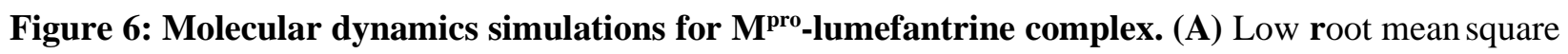
fluctuations of $\mathrm{C} \alpha$ protein atoms suggest protein was stable during the simulation run. (B) The stable RMSD of $\mathrm{C} \alpha$ protein atoms (Blue) and Ligand RMSD (Red) suggests formation of stable protein-ligand complex. 